\title{
Metastatic skull tumors: MRI features and a new conventional classification
}

\author{
Koichi Mitsuya $\cdot$ Yoko Nakasu $\cdot$ Satoshi Horiguchi $\cdot$ \\ Hideyuki Harada $\cdot$ Tetsuo Nishimura . \\ Sachiko Yuen · Koiku Asakura • Masahiro Endo
}

Received: 15 August 2010/Accepted: 8 November 2010/Published online: 26 November 2010

(C) The Author(s) 2010. This article is published with open access at Springerlink.com

\begin{abstract}
Skull metastases are malignant bone tumors which are increasing in incidence. The objectives of this study were to characterize the MR imaging features, locations, and extent of metastatic skull tumors to determine the frequency of the symptomatic disease, and to assess patient outcomes. Between September 2002 and March 2008, 175 patients undergoing routine head MR imaging were found to have metastatic skull tumors. Contrast-enhanced study with fat suppression was used in some cases when required. Classification of metastases was simplified to three yes/no questions: first, with regard to location (either in the calvarium or in the cranial base); second, with regard to distribution within the plane of the cranial bone (either "circumscribed" meaning clearly demarcated and confined to one bone, or "diffuse" and likely to spread across a suture to another bone); and third, with regard to invasion ("intraosseous" in cranial bones only, or "invasive" spreading from the skull, either out into the scalp or inward to the dura and perhaps further in). Primary sites were breast cancer $(55 \%)$, lung cancer $(14 \%)$, prostate cancer $(6 \%)$, malignant lymphoma (5\%), and others $(20 \%)$. The mean
\end{abstract}

K. Mitsuya $(\varangle) \cdot$ Y. Nakasu $\cdot$ S. Horiguchi

Division of Neurosurgery, Shizuoka Cancer Center, Sunto, Japan

e-mail: k.mitsuya@scchr.jp

H. Harada $\cdot$ T. Nishimura

Division of Radiation Oncology, Shizuoka Cancer Center,

Sunto, Japan

S. Yuen

Department of Radiology, Kyoto Prefectural University

of Medicine, Kyoto, Japan

K. Asakura · M. Endo

Division of Diagnostic Radiology, Shizuoka Cancer Center,

Sunto, Japan time from primary diagnosis to skull metastasis diagnosis was 71 months for cases of breast cancer, 26 months for prostate cancer, 9 months for lung cancer, and 4 months for malignant lymphoma. Calvarial circumscribed intraosseous metastases were found most frequently (27\%). The patients were mainly asymptomatic. However, some patients suffered from local pain or cranial nerve palsies that harmed their quality of life. Treatment, mainly for symptomatic cases, was by local or whole-skull irradiation. Metastatic skull tumors are not rare, and most are calvarial circumscribed intraosseous tumors. MR images contribute to understanding their type, location, and multiplicity, and their relationship to the brain, cranial nerves, and dural sinuses. Radiation therapy improved the QOL of patients with neurological symptoms.

Keywords Cancer - Metastatic skull tumor - MR image

\section{Introduction}

The cranium is the site of blood-born metastases of various malignancies including carcinoma of the lung, breast, and thyroid, renal cell carcinoma, malignant melanoma in adults, and neuroblastoma in children. Although skull metastases are not rare, and can cause disabling clinical syndromes including pain, they remain neglected complications of systemic malignancies [1]. They are manageable, but early diagnosis is crucial for selecting treatment. Although CT scan is commonly considered appropriate for bone lesion diagnosis, MR images enable screening for metastases in both skull bone and brain. The objective of this study was to present clinical features and outcomes of patients with skull metastases examined in a district cancer center. On the basis of the available data, we suggest 
classifying metastases by their location and by their distribution within and perpendicular to the plane of the bone, as described by three questions answerable by MRI screening.

\section{Subjects and methods}

From September 2002 to March 2008, 175 patients with calvarial and skull-base metastases were identified from the institute date-base. Clinical information was obtained by retrospective chart review, on age, sex, type of primary tumor, presenting symptoms, treatment, systemic metastases, and survival.

Magnetic resonance (MR) images were reviewed to analyze the location, to see whether a tumor invaded any cranial suture, scalp, dura, or brain, and to assess signal intensity and contrast-enhancement features. Any associated intracranial metastases or meningeal dissemination were also recorded.

Imaging procedures

MRI studies were performed with the units Signa 1.5 $\mathrm{T}$ (GE Healthcare) and Intera 1.5 $\mathrm{T}$ (Philips Healthcare). The imaging procedures used, which depended on the condition of the patients, were: T1-weighted images (TR/ TE, 480-530/15, Signa; 408-449/12, Intera), T2-weighted images (3500-3750/102, Signa; 3751-4126/90, Intera), FLAIR images (9000/125, 2,250-ms inversion time, Signa; $6000 / 100,2,000-\mathrm{ms}$ inversion time, Intera), and contrastenhanced T1-weighted images with $0.1 \mathrm{mmol} / \mathrm{kg}$ gadopentetate dimeglumine (Magnevist, Bayer Healthcare). Fat suppression was added to gadolinium-contrast T1-weighted images (430-530/15, Signa; 425-475/12, Intera) when the attending physician requested investigation of bone marrow changes.

Imaging finding and classification

A neuroradiologist and two neurosurgeons evaluated the images. Classification of metastases was simplified to three yes/no questions: first, with regard to location (either in the calvarium or in the cranial base); second, with regard to distribution within the plane of the cranial bone (either "circumscribed", meaning confined to one bone, or "diffuse" and likely to spread across a suture to another bone); and third, with regard to invasion ("intraosseous" or "invasive" spreading out of the bone, either out into the scalp or inward to the dura). Thus each lesion could be described by its location and by its distribution within and perpendicular to the plane of the bone.

Because histologic confirmation of skull metastases was not generally available, diagnosis was based primarily on results from subsequent MR images and other standard diagnostic modalities: CT and radionuclide bone scanning.

No cases of skull-base invasion were included in which a regional primary tumor of the head and neck directly infiltrated along the cranial nerves.

The study protocol was approved by the Institutional Review Board and conducted according to the principles of the Declaration of Helsinki and the rules of Good Clinical Practice.

\section{Results}

Patient characteristics

Table 1 lists the clinical features of 175 patients. The patients' median age was 57 years (range 8-82 years). The ratio of men to women was 3:7. Breast cancer (in 54.9\%) was the most common primary tumor, followed by lung cancer (in 14.3\%), prostate cancer (in 6.3\%), malignant lymphoma (in 5.1\%), and others (in 19.4\%).

Among the primary lung cancer cases, the most common histological types were adenocarcinoma (58\%),

Table 1 Patients' characteristics

\begin{tabular}{lr}
\hline Characteristics & $N=175$ \\
\hline Sex & \\
Women & $122(70 \%)$ \\
Men & $53(30 \%)$ \\
Median age at diagnosis of skull metastases (range) & $57(8-82)$ \\
Primary cancer & \\
Breast & $96(54.9 \%)$ \\
Lung & $25(14.3 \%)$ \\
Prostate & $11(6.3 \%)$ \\
Malignant lymphoma & $9(5.1 \%)$ \\
Gastric & $7(4.0 \%)$ \\
Esophagus & $5(2.9 \%)$ \\
Malignant melanoma & $4(2.3 \%)$ \\
Leukemia & $4(2.3 \%)$ \\
Colon & $2(1.1 \%)$ \\
Liver & $2(1.1 \%)$ \\
Orbit & $1(0.6 \%)$ \\
Thyroid & $1(0.6 \%)$ \\
Thymoma & $1(0.6 \%)$ \\
Intestine & $1(0.6 \%)$ \\
Cartinoid & $1(0.6 \%)$ \\
Uterus cervical & $1(0.6 \%)$ \\
Uterus body & $1(0.6 \%)$ \\
Urinary duct & $1(0.6 \%)$ \\
Bladder & $1(0.6 \%)$ \\
Unknown & $1(0.6 \%)$ \\
\hline
\end{tabular}


followed by small cell carcinoma (18\%), squamous cell carcinoma $(8 \%)$, large cell carcinoma $(8 \%)$, and unknown $(8 \%)$.

Fifty-two (30\%) of all 175 patients had brain parenchymal metastases at their presentation. As for the primary sites, $33 \%$ of patients with lung cancer had both brain and skull metastases, as did $19 \%$ of patients with breast cancer; however, no patient with prostatic cancer had brain parenchymal lesions.

\section{MR features}

Metastases were found in calvarial bones in 121 patients, and in the skull base in 97 patients, so there were 43 patients with both (Table 2).

MR signals varied; there were low to iso-intensity signals on T1-weighted, and low to iso-intensity signals on T2-weighted images. Skull metastases were enhanced by gadolinium to different degrees; most lesions showed nearly homogenous enhancement, but one of the lesions showed only rim enhancement. Examples of calvarial metastases are presented on Fig. 1, and skull-base metastases in Fig. 2.

The distribution of metastases according to the classification described above is shown in Table 2. The most common type was calvarial circumscribed intraosseous metastases (27\%), followed by skull base diffuse invasive $(22 \%)$ then calvarial diffuse invasive metastases (20\%).

Metastases from breast cancer most frequently presented as calvarial circumscribed intraosseous tumors (30 of 96 patients), followed by calvarial diffuse invasive metastases (25 of 96 patients). Metastases from lung cancer (like those from breast cancer) most frequently presented as calvarial circumscribed intraosseous lesions (7 of 24 patients), followed by calvarial circumscribed invasive metastases (6 of 24 patients).

Calvarial circumscribed intraosseous lesions were the commonest skull-bone metastases in cases of both breastcancer (30 of 96 breast-cancer patients) and lung-cancer (7 of 24 lung-cancer patients).

Calvarial diffuse invasive lesions were the secondcommonest skull-bone metastases in cases of lung-cancer (6 of 24 lung-cancer patients).

Calvarial circumscribed invasive lesions were the second-commonest skull-bone metastases in cases of breastcancer (25 of 96 lung-cancer patients).

\section{Clinical manifestations (Table 2)}

Patients with calvarial circumscribed intraosseous metastases had no symptoms. Calvarial circumscribed invasive metastases caused local pain and swelling in five patients (18\%). Four patients (11\%) with calvarial diffuse invasive
Table 2 MR classification and symptoms

\begin{tabular}{lclr}
\hline MR classification & Incidence $(\%)^{\mathrm{b}}$ & Symptom & $\begin{array}{c}\text { Symptomatic } \\
\text { cases }(\%)^{\mathrm{c}}\end{array}$ \\
\hline $\begin{array}{l}\text { Calvaria }\left(N=121^{\mathrm{a}}\right) \\
\text { Circumscribed }\end{array}$ & & & \\
Intraosseous & $27 \%(48 / 175)$ & None & $0 \%(0 / 48)$ \\
Invasive & $22 \%(38 / 175)$ & Pain & $13 \%(5 / 38)$ \\
Diffuse & & Cosmetic problem & $8 \%(3 / 38)$ \\
Intraosseous & $6 \%(10 / 175)$ & None & $0 \%(0 / 10)$ \\
Invasive & $20 \%(30 / 175)$ & Meningeal irritation & $10 \%(3 / 30)$ \\
Skull base $\left(N=97^{\mathrm{a}}\right)$ & & Disorientation & $10 \%(3 / 30)$ \\
Circumscribed & & & $11 \%(2 / 18)$ \\
Intraosseous & $10 \%(18 / 175)$ & Pain & $29 \%(7 / 26)$ \\
Invasive & $15 \%(26 / 175)$ & $\mathrm{CN}$ sign & $15 \%(4 / 26)$ \\
& & Pain & $12 \%(3 / 26)$ \\
Diffuse & & Direct compression on the orbit & \\
Intraosseous & $8 \%(14 / 175)$ & Pain & $21 \%(3 / 14)$ \\
Invasive & $22 \%(39 / 175)$ & $\mathrm{CN}$ sign & $33 \%(13 / 39)$ \\
& & Pain & $10 \%(4 / 39)$ \\
\hline
\end{tabular}


Fig. 1 MR images show four types of calvarial metastasis. a T1-weighted image shows a lesion of low-intensity signal with clear margin;

circumscribed intraosseous type. b T1-weighted image shows an oval lesion of isointensity signal invading to the dura; circumscribed invasive type. c T1-weighted image shows heterogeneous bone marrow; diffuse intraosseous type. d T1-weight image with contrast media shows heterogeneous enhancement of the parietal bone, dura and the cortical sulci; diffuse invasive type
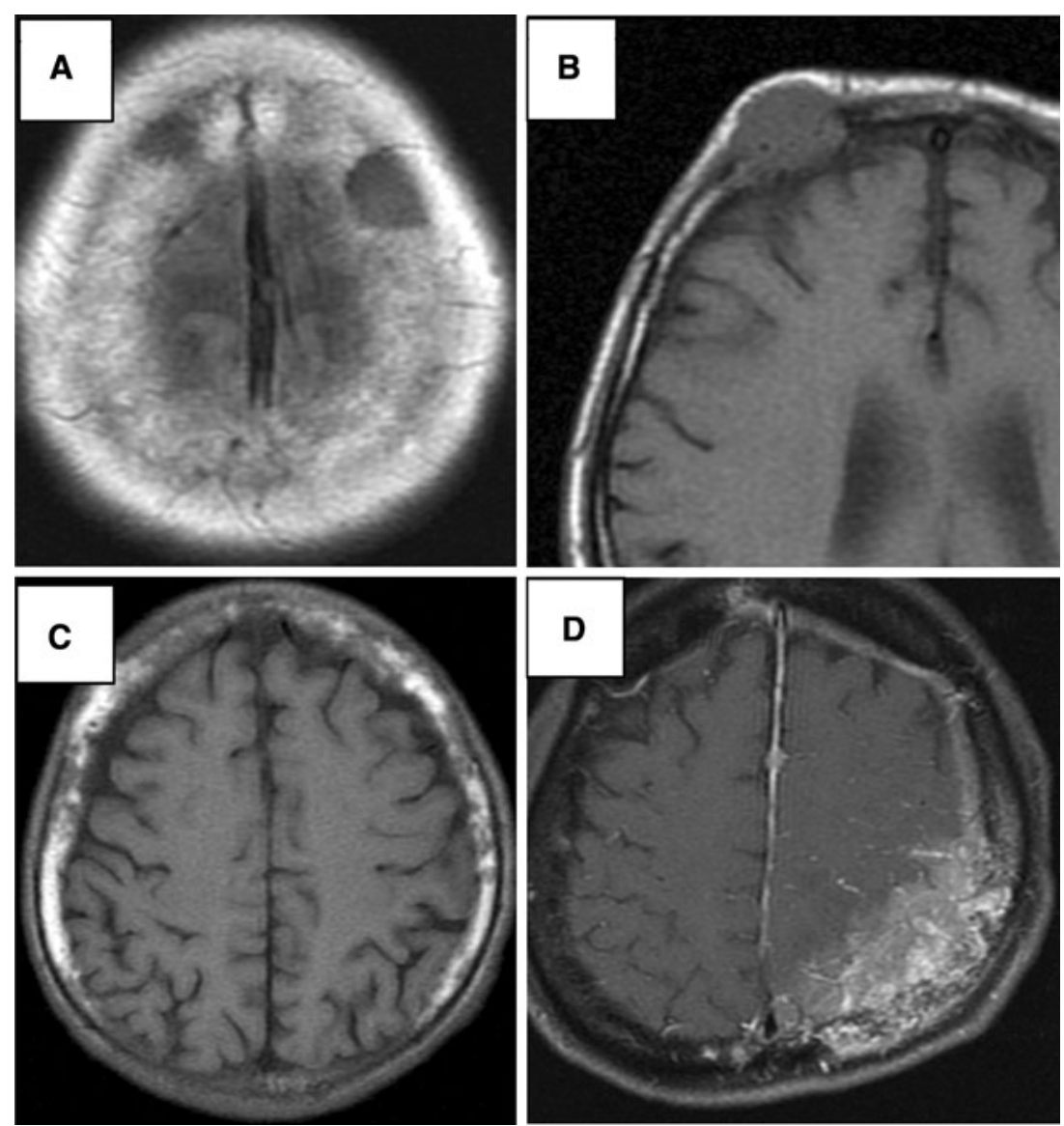

Fig. 2 MR images show four types of skull-base metastasis. a T1-weighted image shows a lesion of low-intensity signal with clear margin in the right pyramid apex; circumscribed intraosseous type. b T1-weighted image with contrast media shows a multilobulated lesion in the sphenoid bone invading the temporal muscle and the dura; circumscribed invasive type. c T1-weighted image with contrast media and fat suppression shows diffuse enhancement of the clivus and pyramis apex; diffuse intraosseous type. d T1-weighted image with contrast media and fat suppression shows

heterogeneous enhancement of the skull base and the dura of the middle fossa; diffuse invasive type
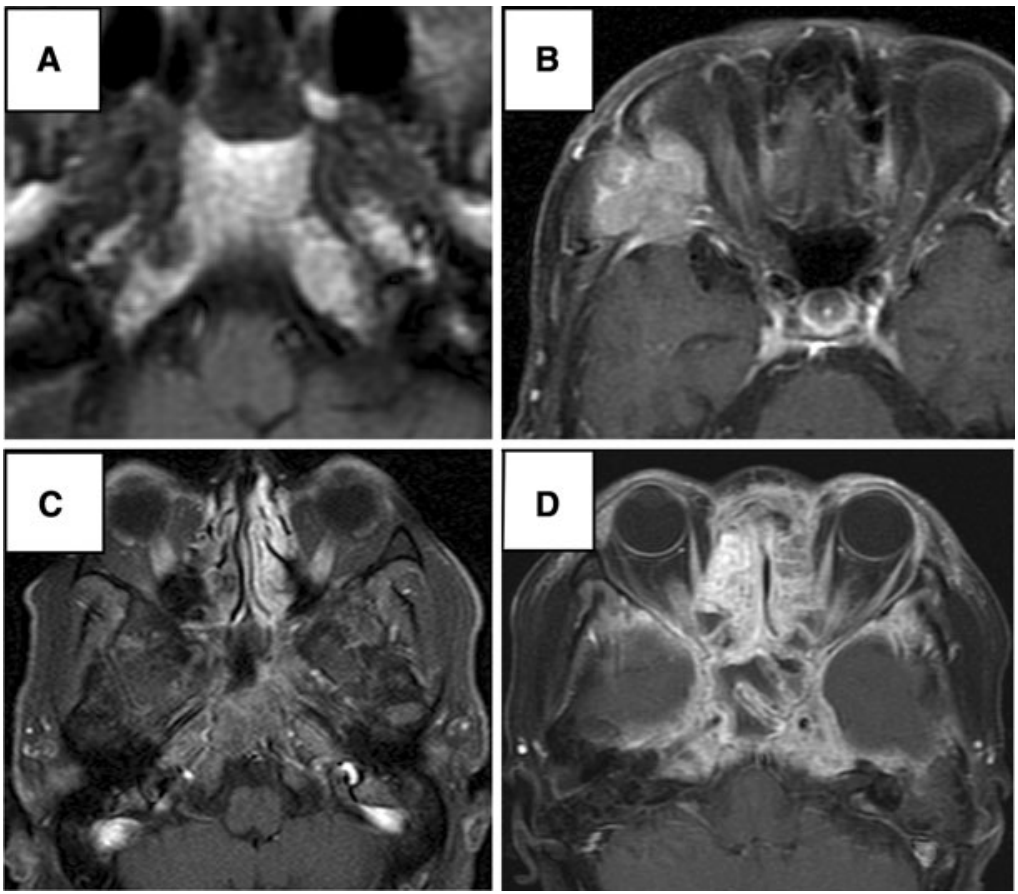

metastases suffered from headache and nausea because of dural and subdural invasion. As demonstrated in Table 2, the most common symptom in our patients with skull-base metastasis was cranial nerve signs (21\%), followed by pain (13\%). Of 20 patients with cranial nerve signs, 11 (55\%) presented with diplopia, $4(20 \%)$ with trigeminal nerve 
Table 3 Primary cancer and intervals from diagnosis of primary malignancy to skull metastases

\begin{tabular}{|c|c|c|c|c|}
\hline \multirow[t]{2}{*}{ Primary tumor } & \multicolumn{2}{|l|}{ This study } & \multicolumn{2}{|c|}{ Greenberg et al. 1981 [2] (skull base only } \\
\hline & No. & Median time $(M$, range $)$ & No. & Median time $(M$, range $)$ \\
\hline Breast & $96(55 \%)$ & $71(1-197)$ & $17(40 \%)$ & $66(2-192)$ \\
\hline Lung & $25(14 \%)$ & $9(0-40)$ & $6(14 \%)$ & $3(0-36)$ \\
\hline Prostate & $11(6 \%)$ & $26(0-132)$ & $5(12 \%)$ & $23(1-39)$ \\
\hline Head and neck & $1(1 \%)$ & $0(0)$ & $6(14 \%)$ & $12(7-72)$ \\
\hline Lymphoma & $9(5 \%)$ & $4(0-8)$ & $3(7 \%)$ & $20(18-32)$ \\
\hline Miscellaneous & $34(19 \%)$ & $7(1-61)$ & $6(14 \%)$ & $18(1-132)$ \\
\hline Total & 175 & $17(0-197)$ & 43 & $23(0-192)$ \\
\hline
\end{tabular}

sign, 4 (20\%) with hypogrossal nerve dysfunction, and 3 (15\%) with facial nerve palsy.

The median interval between diagnosis of the primary tumors and detection of skull metastases was 4 years (mean 6 years; range $0-16$ years 5 months). The mean survival time from skull metastasis in the 175 patients was 19.5 months (median 9 months; range 3-65 months). The time from primary diagnosis to skull metastases was 71 months for cases with breast cancer, 26 months for prostate cancer, 9 months for lung cancer, and 4 months for malignant lymphoma [2] (Table 3).

Treatment

Patients with asymptomatic skull metastases and without brain metastases were followed up with regular MR imaging.

Sixty-three $(36 \%)$ of our patients underwent radiation therapy. Thirty-five (56\%) of those 63 patients had simultaneous brain parenchymal metastases, and needed wholebrain radiation. Another 28 patients were symptomatic because of skull metastases: $23(82 \%)$ patients showed improvement of their symptoms or stable disease after local or whole cranial radiation.

Overall survival from diagnosis of metastatic skull tumor was 23 months for patients with prostate cancer, 15 months for breast cancer, 6 months for malignant lymphoma, and 5 months for lung cancer (Fig. 3).

\section{Discussion}

Incidence and general consideration

Our study showed breast cancer is the most frequent source of skull metastases both for calvarial metastases (of 122 patients) and for skull-base metastases (of 97 patients). This contributed to the large preponderance of female vs male patients (7:3) in our study. We found no significant difference in primary cancer between calvarial and skull-base metastases. There has been no study of large series

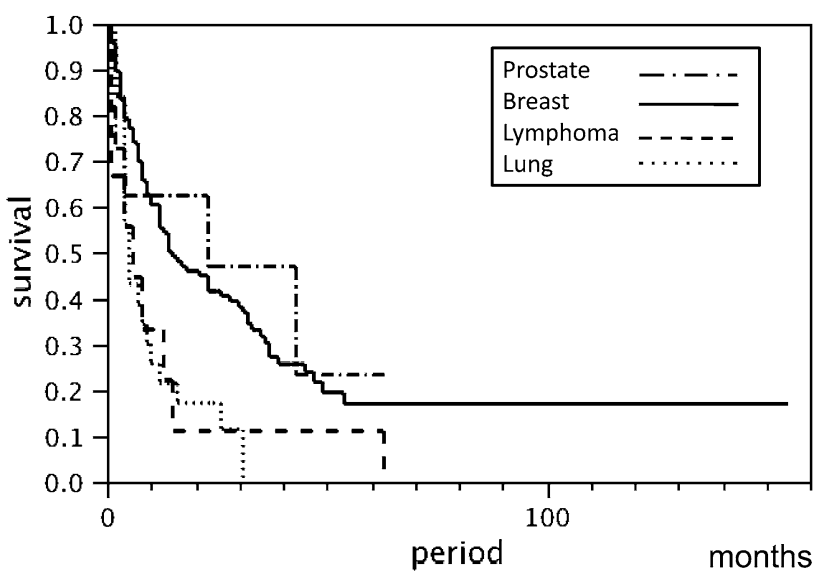

Fig. 3 Kaplan-Meier survival curve of metastatic skull tumors (overall survival from diagnosis of metastatic skull tumors both with and without therapy)

of calvarial metastases. However, a meta-analysis of 279 cases with skull-base metastases demonstrated the most frequent primary tumor was prostate cancer $(38.5 \%)$ followed by breast $(20.5 \%)$ and lymphoma (8\%) [3]. Prostate cancer may cause symptomatic skull-base metastases more frequently than do other malignancies. Also one might overlook skull metastases from prostate cancer, because the patients seldom undergo brain MR imaging for screening.

Sensitivity of imaging

Cancer cells may metastasize to bone marrow, where MR imaging is very sensitive. The normal pattern of fat distribution in the diploic space and marrow of the skull base was symmetric. Gross asymmetry of the diploic space alone is highly suggestive of calvarial disease on precontrast and post-contrast images. MRI using T2 and T1-weighted sequences before and after intravenous gadolinium administration is the best way to detect skull metastases. Fat suppression in combination with gadolinium infusion is particularly important. The main finding in skull metastases consists of substitution of the usual 
hyperintense fat sign by a hypointense lesion on nonenhanced T1-weighted images with a variable appearance on T2-weighted images. T1-weighted sequences with fat suppression show variable enhancement after gadolinium infusion [3]. MR is particularly useful to show any invasion into the dura or cranial nerves. Careful comparison of T2-weighted, T1-weighted, and enhanced images is crucial for detection of subtle intradiploic metastases.

A normal adult skull base shows low and high-intensity signals mixed in T1-weighted images and iso-intensity with the pons in T2-weighted images. Typical MR findings of skull-base lymphoma are iso-intense with the grey matter on T1-weighted images, but giving high-intensity signal on T2-weighted images, and homogenous gadolinium enhancement $[4,5]$.

CT-scan with bone windows is a useful method to show lytic bone lesions. However, CT scan does not clearly show boundaries and degrees of dural invasion by bone metastasis. It has poorer spatial resolution for concomitant brain metastasis than does MR imaging.

Radionuclide bone scan offers relatively poor sensitivity in detecting purely osteolytic bone metastases [6], because this technique requires enhanced remodeling of bone to become positive. Also, bone scintigraphy may be negative when MR image is positive [7].

FDG-PET has become an important oncological technique. Diagnostic accuracy of bone metastasis was comparable with that of PET and bone scintigram in a recent study [6]. However, calvarium is usually intentionally excluded from FOV of FDG-PET scan setting because of the large amount of glucose in the normal cranium.

\section{Classification of the skull metastases}

The three-question classification which we propose here can facilitate early identification and diagnosis of metastases on checking head MR images that are taken during routine screening or assessment of brain metastasis. Invasive type metastases in particular must be carefully followed up, even if they are asymptomatic.

\section{Clinical presentation and symptoms}

Metastatic skull tumors are clinically important for patients' quality of life, although they may not directly influence survival time. Calvarial metastases may cause superficial focal pain and cosmetic problems [8], and once calvarial metastases invade into the dura and intradural space, patients suffer from increased intracranial pressure, meningeal irritation, and focal neurological signs.

Skull-base metastases usually cause various combinations of cranial nerve signs. Lower cranial nerve involvements are serious clinical manifestations, sometimes
Table 4 Clinical syndromes associated with skull-base metastases

\begin{tabular}{lcc}
\hline Clinical syndrome & This study & $\begin{array}{l}\text { Greenberg } \\
\text { et al. [2] }\end{array}$ \\
\hline Orbital syndrome & $4(20 \%)$ & $3(7 \%)$ \\
Parasellar syndrome & $7(35 \%)$ & $7(16 \%)$ \\
Middle fossa (Gasserian ganglion) syndrome & $4(20 \%)$ & $15(35 \%)$ \\
Jugular foramen syndrome & $3(15 \%)$ & $9(21 \%)$ \\
Occipital condyle syndrome & $2(10 \%)$ & $9(21 \%)$ \\
Total & 20 & 43 \\
\hline
\end{tabular}

causing aspiration pneumonia. Five syndromes enable convenient understanding of the anatomical features of skull-base tumors (Table 4). The five syndromes listed in Table 4 illustrate the clinical features of skull-base tumors [2]. Among those five syndromes, occipital condyle syndrome is of particular importance in differential diagnosis for patients with unilateral severe occipital headache. Cancers can metastasize to the condyle, causing severe pain and deviation of the protruded tongue toward the side of the pain; metastases from prostatic, colon, and other cancers have recently been reported [9, 10]. Initial imaging may be within normal limits in occipital condyle syndrome, however, patients should be followed up closely [9]. In our series, orbital and parasellar syndromes were frequently seen, although other syndromes were rare.

\section{Treatment of skull metastases}

Four modalities were used for patients with skull metastasis: irradiation, chemotherapy, endocrinological therapy, and surgical removal.

Conventional fractionated radiation therapy remains the primary treatment. Vikram and Chu reported that radiation had better effects for patients presenting with symptoms of short duration; response rate was $87 \%$ for patients with less than 1 month history, $69 \%$ for patients with 1-3 months history, however, $25 \%$ for those with $>3$ months history [11].

Radiosurgery provides good tumor control, with side effects comparable with those of standard radiation therapy [2]. Patients with larger lesions near sensitive structures or in a previously irradiated field might benefit from the fractionated stereotactic radiation technique.

Small-cell lung carcinomas, breast cancers, and prostate cancers are sensitive to radiation therapy and chemotherapy or hormone therapy. Renal cell carcinoma or melanoma is relatively radio-resistant.

Only a minority of patients with skull metastases are candidates for surgical resection. Michael et al. reported that 13 patients with metastases overlying dural sinus underwent surgical resection [12]. Renal cell carcinoma 
and sarcoma were the most common primary malignancies. Overall median actual survival was 16.5 months.

Recent advances in chemotherapy have contributed in treatment of metastatic skull tumors and systemic bone metastasis. Bisphosphonates (BPs) are effective as a palliative treatment for painful bone metastasis and other bone complications [13]. Zoledronate, a BP, has been increasingly used for patients because of improved convenience in clinical application. However, long-term treatment with BPs may cause mandibular necrosis; patients are advised to consult an oral surgeon before undergoing treatment with BPs [14]. Other new bone-specific medications which may contribute to improving clinical manifestations and survival are an isotope ${ }^{89} \mathrm{Sr}$, and an antibody for receptor activator of NF-kappa B (RANKL), denosumab [15].

\section{Conclusion}

Skull metastases can be diagnosed by use of routine head MR images acquired for screening or assessing brain metastases. Here we have proposed a simple system of classification for succinct and clear description of those lesions. Early diagnosis then treatment with irradiation can give patients a better quality of life.

Acknowledgment The authors would like to thank Mr Piers Vigers for constructive discussion and editorial support.

Conflict of interest None declared.

Open Access This article is distributed under the terms of the Creative Commons Attribution Noncommercial License which permits any noncommercial use, distribution, and reproduction in any medium, provided the original author(s) and source are credited.

\section{References}

1. De Monte F, Hanbali F, Ballo MT (2005) Skull base metastasis. In: Berger MS, Prados MD (eds) Text book of neuro-oncology. Elsevier, Philadelphia, pp 466-475

2. Greenberg HS, Deck MD, Vikram B (1981) Metastasis to the base of the skull: clinical findings in 43 patients. Neurology $31: 530-537$
3. Laigle-Donadey F, Taillibert S, Martin-Duverneuil N, Hildebrand J, Delattre JY (2005) Skull-base metastases. J Neurooncol 75:63-69

4. Kimura F, Kim KS, Friedman H, Russell EJ, Breit R (1990) MR imaging of the normal and abnormal clivus. Am J Neuroradiol 11:1015-1021

5. Jung CS, Zimmermann M, Seifert V (2004) Clivus lymphoma. Acta Neurochir 146:533-534

6. Fujimoto R, Higashi T, Nakamoto Y, Hara T, Lyshchik A, Ishizu K, Kawashima H, Kawase S, Fujita T, Saga T, Togashi K (2006) Diagnostic accuracy of bone metastases detection in cancer patients: comparison between bone scintigraphy and whole-body FDG-PET. Ann Nucl Med 20:399-408

7. Haubold-Reuter BG, Duewell S, Schilcher BR, Marincek B, von Schulthess GK (1993) The value of bone scintigraphy, bone marrow scintigraphy and fast spin-echo magnetic resonance imaging in staging of patients with malignant solid tumours: a prospective study. Eur J Nucl Med 20:1063-1069

8. Nayak L, Abrey LE, Iwamoto FM (2009) Intracranial dural metastases. Cancer 115:1947-1953

9. Moeller JJ, Shivakumar S, Davis M, Maxner CE (2007) Occipital condyle syndrome as the first sign of metastatic cancer. Can J Neurol Sci 34:456-459

10. Capobianco DJ, Brazis PW, Rubino FA, Dalton JN (2002) Occipital condyle syndrome. Headache 42:142-146

11. Vikram B, Chu FC (1979) Radiation therapy for metastases to the base of the skull. Radiology 130:465-468

12. Michael CB, Gokaslan ZL, DeMonte F, McCutcheon IE, Sawaya R, Lang FF (2001) Surgical resection of calvarial metastases overlying dural sinuses. Neurosurgery 48:745-754

13. Rosen LS, Gordon D, Tchekmedyian NS, Yanagihara R, Hirsh V, Krzakowski M, Pawlicki M, De Souza P, Zheng M, Urbanowitz G, Reitsma D, Seaman J (2004) Long-term efficacy and safety of zoledronic acid in the treatment of skeletal metastases in patients with nonsmall cell lung carcinoma and other solid tumors: a randomized, phase III, double-blind, placebo-controlled trial. Cancer 100:2613-2621

14. Bamias A, Kastritis E, Bamia C, Moulopoulos LA, Melakopoulos I, Bozas G, Koutsoukou V, Gika D, Anagnostopoulos A, Papadimitriou C, Terpos E, Dimopoulos MA (2005) Osteonecrosis of the jaw in cancer after treatment with bisphosphonates: incidence and risk factors. J Clin Oncol 23:8580-8587

15. Iguchi $H$ (2007) Molecular mechanism and potential targets for bone metastasis. Gan To Kagaku Ryoho 34:1-10 